Journal of Mathematics and Statistics 8 (1): 150-153, 2012

ISSN 1549-3644

C 2012 Science Publications

\title{
Weyl's Theorem for Algebraically Class A(s, t) Operators
}

\author{
Senthilkumar, D. and D. Kiruthika \\ Department of Mathematics, \\ Government Arts College (Autonomous),Coimbatore-641 018, Tamil Nadu, India
}

\begin{abstract}
In this study, we show that Weyl's theorem holds for algebraically class A (s, t) operator acting on Hilbert space. We prove: (i) Weyl's theorem holds for $f(T)$ for every $f$ belongs to holomorphic function of spectrum of T; (ii) generalized Weyl's theorem holds for T; (iii) the spectral mapping theorem holds for the Weyl spectrum of $\mathrm{T}$ and for the essential approximate point spectrum of $\mathrm{T}$.
\end{abstract}

Key words: Class A (s, t), SVEP, isoloid, Weyl's theorem

\section{INTRODUCTION}

Let $\mathrm{H}$ be an infinite dimensional Hilbert space and $\mathrm{B}(\mathrm{H})$ denote the algebra of all bounded linear operator acting on $\mathrm{H}$. Let $\mathrm{T}$ be an operator whose polar decomposition is $\mathrm{T}=\mathrm{U}|\mathrm{T}|$, where $|\mathrm{T}|=\sqrt{\mathrm{T}^{*} \mathrm{~T}}$. An operator $\mathrm{T}$ is said to be p-hyponormal, for $\mathrm{p} \in(0,1)$, if $\left(\mathrm{T}^{*} \mathrm{~T}\right)^{\mathrm{p}}$ $\geq\left(\mathrm{TT}^{*}\right)^{\mathrm{p}}$ by A. Aluthge. A 1-hyponormal operator is hyponormal and $\frac{1}{2}$ - hyponormal operator is said to be semi-hyponormal. An invertible operator $\mathrm{T}$ is said to be $\log$ - hyponormal if $\log |\mathrm{T}| \geq \log \left|\mathrm{T}^{*}\right|$ (Tanahashi., 1999). An operator $\mathrm{T}$ is said to be paranormal if $\left\|\mathrm{T}^{2} \mathrm{x}\right\| \geq\|\mathrm{Tx}\|^{2}$ for every unit vector $\mathrm{x}$. As a generalization of a class $\mathrm{A}$ and paranormal operators they introduced class $\mathrm{A}(\mathrm{k})$ and absolute k-paranormal operator respectively (Furuta et al., 1998). An operator T belongs to class A (k) for $\mathrm{k}>0$ if $\left(\mathrm{T}^{*}|\mathrm{~T}|^{2 \mathrm{k}} \mathrm{T}\right)^{\frac{1}{\mathrm{k}+1}} \geq|\mathrm{T}|^{2}$ an absolute $\mathrm{k}$ paranormal if $\left\||T|^{k} T x\right\| \geq\|T x\|^{k+1}$ for every unit vector $x \in H$. It has been proved that every $\log$ hyponormal operator is class $A(k)$, every class $A(k)$ operator is absolute k-paranormal (Furuta et al.,1998). When $\mathrm{k}=1$ we say that $\mathrm{T}$ belongs to class $\mathrm{A}$ operator.

As a further generalization of class A (k), (Masatoshi et al., 2000) introduced the class $\mathrm{A}(\mathrm{s}, \mathrm{t})$. An operator $\mathrm{T}$ belongs to class $\mathrm{A}(\mathrm{s}, \mathrm{t})$, for $\mathrm{s}>0$ and $\mathrm{t}>0$ if $\left.\left(\left.\mathrm{T}^{*}\right|^{\mathrm{t}}|\mathrm{T}|^{2 \mathrm{~s}}\left|\mathrm{~T}^{*}\right|^{\mathrm{t}}\right)^{\mathrm{t}} \mathrm{ts} \mathrm{T} \mathrm{T}^{*}\right|^{2 \mathrm{t}}$. In other words class $\mathrm{A}(\mathrm{s}, \mathrm{t})$ operator is defined as $|\mathrm{T}(\mathrm{s}, \mathrm{t})|^{\frac{2 \mathrm{t}}{\mathrm{s}+\mathrm{t}}} \geq|\mathrm{T}|^{2 \mathrm{t}}$. Many others have studied its properties in (Yamazaki et al., 1999; Ito and
Yamazaki, 2002; Uchiyama, 2001) and (Uchiyama and Tanahashi, 2002). It is known that p-hyponormal operators and log - hyponormal operators are class $\mathrm{A}(\mathrm{s}$, t) operators and $\mathrm{T}(\mathrm{s}, \mathrm{t})$ is $\frac{\min \mathrm{s}, \mathrm{t}}{\mathrm{s}+\mathrm{t}}$ - hyponormal for all $0<s$, t. If $T$ is a class $A(s, t)$ operator and $s \leq s^{\prime}, t \leq t^{\prime}$, then $\mathrm{T}$ is a class $\mathrm{A}\left(\mathrm{s}^{\prime}, \mathrm{t}^{\prime}\right)$. An operator $\mathrm{T}$ is a class $\mathrm{A}(1$, 1) operator if and only if $T$ is a class $A$ operator (Masatoshi et al., 2000; Yamazaki et al., 1999; Wang and Lee, 2003; Ito, 1999; Ito and Yamazaki, 2002; Tanahashi, 1999; Yoshino, 1997). Class AI (s, t) is the class of all invertible class $\mathrm{A}(\mathrm{s}, \mathrm{t})$ operator for $s>0$ and $t>0$. It was pointed out in (Yanagida, 2000) that class $A(k, 1)$ equals class $A(k)$. They showed several properties of class A (s, t) and class AI (s, t) as extensions of the properties of class A (k) shown in (Yamazaki et al., 1999). Spectral properties of class A $(s, t)$ operators where $s, t \in(0,1)$ have been studied by several authors ((Uchiyama and Tanahashi, 2002, Uchiyama et al., 2004). The spectral properties of class A $(s, t)$ operators where $\mathrm{s}>1, \mathrm{t}>1$ via their generalized Aluthge transformation and hyponormal transforms has been studied by Stella. An operator $\mathrm{T}$ is said to be of algebraically class A (s, $\mathrm{t}$ ), if there exists a non-constant complex polynomial $\mathrm{p}$ such that $\mathrm{p}(\mathrm{T})$ is of class A (s, t) operator.

\section{Preliminaries:}

Lemma 1: (Rashid and Zguitti, 2011): Let $T$ belongs to the class A (s, t) for some $0<\mathrm{s}, \mathrm{t} \leq 1, \lambda \in \mathrm{C}$ and assume that $\sigma(\mathrm{T})=\lambda$. Then $\mathrm{T}=\lambda$.

Lemma 2: Let $\mathrm{T}$ be invertible and quasi nilpotent algebraically class A $(\mathrm{s}, \mathrm{t})$ operator. Then $\mathrm{T}$ is nilpotent. 
Proof: Suppose that $\mathrm{p}(\mathrm{T})$ is class A $(\mathrm{s}, \mathrm{t})$ operator for some non-constant polynomial p. Since $\sigma(\mathrm{p}(\mathrm{T}))=$ $p(\sigma(T))$, the operator $p(T)-p(0)$ is quasi-nilpotent, from Lemma 1 we have:

$$
\begin{aligned}
& \mathrm{CT}^{\mathrm{m}}\left(\mathrm{T}-\lambda_{1}\right)\left(\mathrm{T}-\lambda_{2}\right) \ldots \ldots \ldots \ldots \ldots \ldots \ldots \\
& \left(\mathrm{T}-\lambda_{\mathrm{n}}\right) \equiv \mathrm{p}(\mathrm{T})-\mathrm{P}(0)=0
\end{aligned}
$$

where, $\mathrm{m} \geq 1$ since $\mathrm{T}-\lambda_{\mathrm{i}}$ is invertible for every $\lambda_{\mathrm{I}} \neq 0$ and so $\mathrm{T}_{\mathrm{m}}=0$.

Lemma 3: Let $\mathrm{T}$ be an algebraically class $\mathrm{A}(\mathrm{s}, \mathrm{t})$ operator. Then $\mathrm{T}$ is isoloid.

Proof: Let $\lambda \in \operatorname{is} \sigma(T)$ and let $E_{\lambda}=\frac{1}{2 \pi i} \int_{\partial \mathrm{D} \lambda}(Z-T)^{-1} d z$ be the associated Riesz idempotent, where $\mathrm{D}_{\lambda}$ is a closed disc centered at $\lambda$ which contains no other point of $\sigma$ (T). We can represent $\mathrm{T}$ as the direct sum, $\mathrm{T}=\left(\begin{array}{cc}\mathrm{T}_{1} & 0 \\ 0 & \mathrm{~T}_{2}\end{array}\right)$ where $\sigma\left(\mathrm{T}_{1}\right)=\lambda$ and $\sigma\left(\mathrm{T}_{2}\right)=\sigma(\mathrm{T}) \lambda$. Since Tis algebraically class $A(s, t)$ operator $p(T)$ is a class $A(s$, t) operator for some non-constant polynomial $\mathrm{p}$. Since $\sigma\left(\mathrm{T}_{1}\right)=\lambda$, we must have $\sigma\left(\mathrm{p}\left(\mathrm{T}_{1}\right)\right)=\mathrm{p}\left(\sigma\left(\mathrm{T}_{1}\right)=\mathrm{p}(\lambda)\right.$. Therefore $\left(\mathrm{p}\left(\mathrm{T}_{1}\right)\right)-\mathrm{p}(\lambda)$ is quasi-nilpotent.

Since $\mathrm{p}\left(\mathrm{T}_{1}\right)$ is class $A(s, t)$ operator, it follows from Lemma 1 , that $\mathrm{p}\left(\mathrm{T}_{1}\right)-\mathrm{p}(\lambda)=0$. Put. Then $\mathrm{q}\left(\mathrm{T}_{1}\right)$ $=0$ and hence $T_{1}$ is algebraically class $A(s, t)$ operator. Since $T_{1}-\lambda$ is quasi-nilpotent and algebraically class $A$ $(s, t)$ operator, it follows from Lemma 2 , then $T_{1}-\lambda$ is nilpotent. Therefore $\lambda \in \pi_{0}(\mathrm{~T})$ and hence $\lambda \in \pi_{0}(\mathrm{~T})$. This proves that $\mathrm{T}$ is isoloid.

Theorem 4 (Rashid and Zguitti, 2011): Let $\mathrm{T}$ belongs to the class $A(s, t)$ for some $0<s, t \leq 1$. Then $T$ is of finite ascent.

Corollary 5 (Rashid and Zguitti, 2011): Let $T$ belongs to the class $A(s, t)$ for some $0<s, t \leq 1$. Then $T$ has SVEP.

Theorem 6: Let $\mathrm{T}$ be an algebraically class $\mathrm{A}(\mathrm{s}, \mathrm{t})$ operator. Then T has SVEP.

Proof: First we show that if $\mathrm{T}$ is class $\mathrm{A}(\mathrm{s}, \mathrm{t})$ operator, then $\mathrm{T}$ has SVEP. Suppose that $\mathrm{T}$ is class $\mathrm{A}(\mathrm{s}, \mathrm{t})$ operator. If $\pi_{0}(T)=\varphi$, then clearly $T$ has SVEP. Suppose that $\pi_{0}(T) \neq \varphi$. Let $\Delta(T)=\lambda \in \pi_{0}(T): \quad N(T-$ $\lambda) \subseteq N\left(T^{*}-\bar{\lambda}\right)$. Since $\mathrm{T}$ is class $\mathrm{A}(\mathrm{s}, \mathrm{t})$ operator and $\pi_{0}(\mathrm{~T}) \neq \varphi, \Delta(\mathrm{T}) \neq \varphi$ Let $\mathrm{M}$ be the closed linear span of the subspaces $\mathrm{N}(\mathrm{T}-\lambda)$ with $\lambda \in \Delta(\mathrm{T})$. Then $\mathrm{M}$ reduces $\mathrm{T}$ and we can write $\mathrm{T}$ as $\mathrm{T}_{1} \oplus \mathrm{T}_{2}$ on $\mathrm{H}=\mathrm{M} \oplus \mathrm{M}^{\perp}$. Clearly $T_{1}$ is normal and $\pi_{0}\left(T_{2}\right)=\varphi$. Since $T_{1}$ and $T_{2}$ have both SVEP, $T$ has SVEP. Suppose that $T$ is algebraically class A $(s, t)$ operator. Then $p(T)$ is class A $(s, t)$ operator for some non constant polynomial $p$. Since $p(T)$ has SVEP, it follows from [Laursen and Neumann., 2000, Theorem 3.3.9] that T has SVEP.

\section{RESULTS AND DISCUSSION}

Weyl's theorem for algebraically class $\mathbf{A}(\mathbf{s}, \mathbf{t})$ operators: Let $T \in B(H)$, we write $N(T)$ and $R(T)$ for the null space and range of $\mathrm{T}$ respectively. Let $\alpha(\mathrm{T})=$ $\operatorname{dim} N(\mathrm{~T})$ and $\beta(\mathrm{T})=\operatorname{dim} N\left(\mathrm{~T}^{*}\right)$ where $\mathrm{T}^{*}$ is the adjoint of space of T. Let $\sigma(\mathrm{T}), \sigma_{\mathrm{p}}(\mathrm{T}), \sigma_{\alpha}(\mathrm{T})$ and $\pi_{0}$ $(\mathrm{T}), \pi_{00}(\mathrm{~T})$ denote the spectrum, point spectrum, approximate point spectrum, the set of eigen values of $\mathrm{T}$ of finite multiplicity and isolated points of $\sigma(\mathrm{T})$. An operator $\mathrm{T} \in \mathrm{B}(\mathrm{H})$ is called Fredholm if it has closed range, finite dimensional null space and its range has finite co-dimensional. The index of a Fredholm operator $\mathrm{T} \in \mathrm{B}(\mathrm{H})$ is given by:

$$
\operatorname{ind}(\mathrm{T})=\operatorname{dim} \mathrm{N}(\mathrm{T})-\operatorname{dim} \mathrm{R}(\mathrm{T})^{\perp}=\left(\operatorname{dim} \mathrm{N}(\mathrm{T})-\operatorname{dim} \mathrm{N}\left(\mathrm{T}^{*}\right)\right)
$$

An operator $\mathrm{T} \in \mathrm{B}(\mathrm{H})$ is called Weyl if it is Fredholm of index zero. An operator $T \in B(H)$ is called Browder if it is Fredholm of "finite ascent and decent" equivalently (Harte, 1988) if $\mathrm{T}$ is Fredholm and $\mathrm{T}-\lambda_{\mathrm{i}}$ is invertible for sufficiently small $\lambda \neq 0$ in $C$. The essential spectrum $\sigma_{p}(T)$, the Weyl spectrum $w(T)$ and the Browder spectrum $\sigma_{b}(T)$, of $T \in B(H)$ are defined in (Harte, 1985; 1988).

Theorem 7: Let $\mathrm{T}$ be an algebraically class $\mathrm{A}(\mathrm{s}, \mathrm{t})$ operator. Then Weyl's theorem holds for T.

Proof: Suppose that $\lambda \in \sigma(\mathrm{T}) \backslash \mathrm{w}(\mathrm{T})$. Then $\mathrm{T}=\lambda$ is Weyl and not invertible, we claim that $\lambda \in \partial \sigma(\mathrm{T})$. Assume that $\lambda$ is an interior point of $\sigma(\mathrm{T})$. Then there exist a neighborhood $U$ of $\lambda$, such that $\operatorname{dim} N(T-\mu)>0$ for all $\mu \in \mathrm{u}$. It follows from [Finch, 1975 Theorem 10] that $\mathrm{T}$ does not have SVEP [single valued extension property]. On the other hand, since $\mathrm{p}(\mathrm{T})$ is class $\mathrm{A}(\mathrm{s}, \mathrm{t})$ operator for some non constantpolynomial $\mathrm{p}$, it follows from Lemma 6 , that $\mathrm{T}$ has SVEP. It is a contradiction, Therefore $\lambda \in \partial \sigma(\mathrm{T}) \backslash \mathrm{w}$ (T) and it follows from the punctured neighborhood theorem that $\lambda \in \pi_{00}(\mathrm{~T})$.

Conversely suppose that $\lambda \in \pi_{00}(\mathrm{~T})$. Using the Riesz idempotent $\mathrm{E}_{\lambda}=\frac{1}{2 \pi \mathrm{i}} \int_{\partial \mathrm{D} \lambda}(\mathrm{Z}-\mathrm{T})^{-1} \mathrm{dz}$ for $\lambda$ we can represent 
$\mathrm{T}$ as the direct sum $\mathrm{T}=\left(\begin{array}{cc}\mathrm{T}_{1} & 0 \\ 0 & \mathrm{~T}_{2}\end{array}\right)$ where $\sigma(\mathrm{T})=\lambda$ and $\sigma$ $\left(T_{2}\right)=\sigma(T) \backslash \lambda$.

Now we consider two cases: case (i) $\lambda=0$.

Then $T_{1}$ is algebraically class $A(s, t)$ operator and quasi nilpotent. It follows from Lemma 2 , that $T_{1}$ is nilpotent. We claim that $\operatorname{dim} \mathrm{R}(\mathrm{E})<\propto$. For if $\mathrm{N}\left(\mathrm{T}_{1}\right)$ is infinite dimensional, then 0 does not belongs to $\pi_{00}(\mathrm{~T})$. It is contradiction. Therefore $T_{1}$ is an operator on the finite dimensional space $\mathrm{R}(\mathrm{E})$. So it follows that $\mathrm{T}_{1}$ is Weyl. But since $T_{2}$ is invertible, we can conclude that $T$ is Weyl therefore $0 \in \sigma(T) \backslash w(T)$.

Case (ii) $\lambda \neq 0$ Then by Theorem $3, \mathrm{~T}_{1}-\lambda$ is nilpotent. Since $\lambda \in \pi_{00}(T), T_{1}-\lambda$ is an operator on the finite dimensional space $\mathrm{R}(\mathrm{E})$. So $\mathrm{T}_{1}-\lambda$ is Weyl. Since $\mathrm{T}_{2}-\lambda$ is invertible, $\mathrm{T}-\boldsymbol{\lambda}$ is Weyl.

By case (i) and case (ii), Weyl's theorem holds for T.This completes the proof.

Theorem 8: Let $\mathrm{T}$ be an algebraically class $\mathrm{A}(\mathrm{s}, \mathrm{t})$ operator. Then Weyl's theorem holds for $\mathrm{f}(\mathrm{T})$ forevery $\mathrm{f} \in \mathrm{H}(\sigma(\mathrm{T}))$.

Proof: Let $f \in H(\sigma(T))$. Since $w(f(T)) \subseteq f(w(T))$, it suffices to show that $\mathrm{f}(\mathrm{w}(\mathrm{T})) \subseteq \mathrm{f}(\mathrm{w}(\mathrm{T}))$. Suppose $\lambda \notin \mathrm{w}(\mathrm{f}(\mathrm{T}))$, then $\mathrm{f}(\mathrm{T})-\lambda$ is Weyl and Eq. 1 :

$\mathrm{f}(\mathrm{T})-\lambda=\mathrm{C}\left(\mathrm{T}-\alpha_{1}\right)\left(\mathrm{T}-\alpha_{2}\right) \ldots \ldots \ldots \ldots\left(\mathrm{T}-\alpha_{\mathrm{n}}\right) \mathrm{g}(\mathrm{T})$

where, $\mathrm{C}, \alpha 1, \alpha 2, \ldots \ldots \ldots, \alpha \in \mathrm{C}$ and $\mathrm{g}(\mathrm{T})$ is invertible. Since the operators in the rightside of (1) commute, every $\mathrm{T}-\alpha_{\mathrm{i}}$ is fredholm. Since T is algebraically class A $(s, t)$ operator. T has SVEP by Lemma 6 . It follows from (Aiena and Monsalve, 2000. Theorem 2.6] that ind $\left(\mathrm{T}-\alpha_{\mathrm{i}}\right) \leq 0$ for each $\mathrm{i}=1,2,3, \ldots \ldots . \mathrm{n}$. Therefore $\lambda \notin \mathrm{f}$ $(\mathrm{T}))$ and hence $\mathrm{f}(\mathrm{w}(\mathrm{T}))=(\mathrm{w}(\mathrm{T}))$.

Now by (Lee and Lee, 1996), that is $\mathrm{T}$ is isoloid, then $\left(\sigma(f(T)) \backslash \pi_{00}(T)\right)=\sigma(f(T)) \backslash \pi_{00}(T)$ for every $f \in H$ $(\sigma(T))$. Since $T$ is isoloid by Theorem 3 and Weyl's theorem holds for $\mathrm{T}$ by Theorem $7 \sigma(\mathrm{f}(\mathrm{T}))\left(\pi_{00}(\mathrm{~T})=\mathrm{f}\right.$ $(\sigma(\mathrm{T}))\left(\pi_{00}(\mathrm{~T})=\mathrm{f}(\mathrm{w}(\mathrm{T}))=\mathrm{w}(\mathrm{f}(\mathrm{T}))\right.$ which implies that Weyl's theorem holds for $\mathrm{f}(\mathrm{T})$. This completes the proof.

Theorem 9: Let $\mathrm{T}$ be an algebraically class $\mathrm{A}(\mathrm{s}, \mathrm{t})$ operator. Then generalized Weyl's theorem holds for (T).

Proof: Assume that $\lambda \in \sigma(T) \backslash \sigma_{\mathrm{Bw}}(\mathrm{T})$. Then $\left(\mathrm{T}-\lambda_{\mathrm{i}}\right)$ is BWeyl and not invertible. We claim that $\lambda \in \partial \sigma(\mathrm{T})$.
Assume to the contrary that $\lambda$ is an interior point of $\sigma$ (T). Then there exists a neighborhood $\mathrm{U}$ of $\lambda$ such that $\operatorname{dim}(\mathrm{T}-\mu)>0$ for all $\mu \in \mathrm{U}$. It follows from [Finch, 1975., Theorem 10] that $\mathrm{T}$ does not have SVEP. On the other hand since $\mathrm{p}(\mathrm{T})$ is class A $(\mathrm{s}, \mathrm{t})$ operator for nonconstant polynomial $\mathrm{p}$. It follows from Lemma 6 that $\mathrm{p}$ (T) has SVEP. Hence by [Laursen and Neumann, 2000, Theorem 3.3.9] T has SVEP, a contradiction. Therefore $\lambda \in \partial \sigma(\mathrm{T})$.Conversely, assume that $\lambda \in \mathrm{E}(\mathrm{T})$, then $\lambda$ is isolated in $\sigma(\mathrm{T})$. From [Koliha,1996, Theorem 7.1], we have $\mathrm{X}=\mathrm{M} \oplus \mathrm{N}$, where $\mathrm{M}, \mathrm{N}$ are closed subspaces of $\mathrm{X}$, $\mathrm{U}=(\mathrm{T}-\lambda \mathrm{I}) \mid \mathrm{N}$ is an invertible operator and $\mathrm{V}=\left.(\mathrm{A}-\lambda \mathrm{I})\right|_{\mathrm{N}}$ is a quasi - nilpotent operator. Since $\mathrm{T}$ is algebraically class $\mathrm{A}(\mathrm{s}, \mathrm{t})$ operator, $\mathrm{V}$ is also algebraically class A $(\mathrm{s}, \mathrm{t})$ operator from Lemma $8, \mathrm{~V}$ is nilpotent. Therefore T- $\lambda \mathrm{I}$ is Drazin invertible [Coburn, 1966, Proposition 19] and Lay, 1970, Corollary 2.2]. By [Berkani, 2002, Lemma 4.1]T- $\lambda \mathrm{I}$ is a B - fredholm operator of index 0 .

Theorem 10: Assume that $\mathrm{T}$ or $\mathrm{T}^{*}$ is algebraically class $\mathrm{A}$ $(\mathrm{s}, \mathrm{t})$. Then $\sigma_{\text {ea }}(\mathrm{f}(\mathrm{T}))=\mathrm{f}\left(\sigma_{\text {ea }}(\mathrm{T})\right)$ for every $\mathrm{f} \in \mathrm{H}(\sigma(\mathrm{T}))$.

Proof: Let $\mathrm{f} \in \mathrm{H} \quad(\sigma(\mathrm{T}))$. It suffices to that $f\left(\sigma_{\text {ea }}(T)\right) \subseteq \sigma_{\text {ea }}(f(T))$ for every $f \in H \quad(\sigma(T))$. Suppose that $\lambda \notin \mathrm{T}\left(\sigma_{\text {ea }}(\mathrm{f}(\mathrm{T}))\right)$. Then $\mathrm{f}(\mathrm{T})-\lambda=\mathrm{C}\left(\mathrm{T}-\alpha_{1}\right)(\mathrm{T}-$ $\left.\alpha_{2}\right) \ldots \ldots \ldots\left(\mathrm{T}-\alpha_{\mathrm{n}}\right) \mathrm{g}(\mathrm{T})$ where $\mathrm{C}, \alpha_{1}, \alpha_{2}, \ldots \ldots \ldots, \alpha_{\mathrm{n}}$ and $\mathrm{g}(\mathrm{t})$ is invertible. If $\mathrm{T}$ is algebraically class $\mathrm{A}(\mathrm{s}, \mathrm{t})$ operator, it follows from [Aiena and Monsalve, 2000, Theorem 2.6] that $\mathrm{i}\left(\mathrm{T}-\alpha_{\mathrm{i}}\right) \leq 0$ for each $\mathrm{i}=1,2,3, \ldots \ldots . . \mathrm{n}$. Therefore $\lambda$ does not belongs to $\mathrm{f}\left(\sigma_{\text {ea }}(\mathrm{T})\right)$ and hence $\left(\sigma_{\text {ea }}(\mathrm{T})\right)=\mathrm{f}\left(\sigma_{\text {ea }}(\mathrm{T})\right)$.

Suppose that $\mathrm{T}^{*}$ is algebraically class $\mathrm{A}(\mathrm{s}, \mathrm{t})$ then $T^{*}$ is SVEP. Since $i\left(T-\alpha_{i}\right) \leq 0$ for each $I=1,2,3 \ldots . n$. $\left(\mathrm{T}-\alpha_{\mathrm{i}}\right)$ is Weyl for each $\mathrm{I}=1,2,3, \ldots \ldots . . \mathrm{n}$. Hence $\lambda \notin \mathrm{f}$ $\left(\sigma_{\text {ea }}(\mathrm{T})\right)$ and so $\left(\sigma_{\text {ea }}(\mathrm{T})\right)=\mathrm{f}\left(\sigma_{\text {ea }}(\mathrm{T})\right)$.

This completes the proof.

In this study we discuss Weyls theorem holds for Class A $(\mathrm{s}, \mathrm{t})$.

\section{CONCLUSION}

It can be shown that that Weyl's theorem holds for algebraically class $\mathrm{A}(\mathrm{s}, \mathrm{t})$ operator acting on Hilbert space $\mathrm{H}$. It can also be shown that Weyl's theorem holds for $f(T)$ for every $f \in H(\sigma(T))$ and generalized Weyl's theorem holds for $(\mathrm{T})$. The spectral mapping theorem holds for the Weyl spectrum of $\mathrm{T}$ and for the essential approximate point spectrum of $\mathrm{T}$ is also shown. 


\section{REFERENCES}

Aiena, P. and O. Monsalve, 2000. Operators which do not have the single valued extension property. J. Math Anal., Appli., 250: 435-448. DOI: 10.1006/Jmaa.2000.6966

Berkani, M., 2002. B-Weyl spectrum and poles of the resolvent. J. Math Anal., Appli., 272: 596-603. DOI: $10.1016 / \mathrm{S} 0022-247 \mathrm{X}(02) 00179-8$

Coburn, L.A., 1966. Weyl's theorem for nonnormal operators. Michigan Math. J., 13: 285-288. DOI: $10.1307 / \mathrm{mmj} / 1031732778$

Finch, J.K., 1975. The single valued extension property on a banach space. Pacific J. Math., 58: 61-69.

Furuta, T., T. Yamazaki and M. Yanagida, 1998. On a conjecture related to Furuta-type inequalities with negative powers. Nihonkai Math. J., 9: 213-218.

Harte, R., 1985. Fredholm, weyl and browder theory. Proc. Royal Iris Acad., 85A: 151-176.

Harte, R., 1988. Invertibility and Singularity for Bounded Linear Operators. 1st Edn., M. Dekker New York, ISBN: 0824777549, pp: 590.

Ito, M. and T. Yamazaki, 2002. Relations between two inequalities $\left(B^{\frac{r}{2}} A^{p} B^{\frac{r}{2}}\right)^{\frac{r}{p+r}} \geq B^{r}$ and $B^{p} \geq\left(A^{\frac{p}{2}} B^{r} A^{\frac{p}{2}}\right)^{\frac{p}{p+r}}$ and their applications. Integr. Equ. Oper. Theory, 44: 442-450.

Ito, M., 1999. Some classes of operators associated with generalized aluthge transformation. SUT J. Math., 35: $149-165$.

Koliha, J.J., 1996. A generalized Drazin Inverse. Glasgow Math. J., 38: 367-381.

Laursen, K.B. and M. Neumann, 2000. An Introduction to Local Spectral Theory. 1st Edn., Clarendon Press, Oxford, ISBN: 0198523815, pp: 591.

Lay, D.C., 1970. Spectral analysis using ascent, descent, nullity and defect. Math. Ann., 184: 197214. DOI: $10.1007 / \mathrm{BF} 01351564$
Lee, W.Y. and S.H. Lee, 1996. A spectral mapping theorem for the Weyl spectrum. Glasgow, Math. J. 38: 61-64. DOI: 10.1017/S0017089500031268

Masatoshi, F., J. Dongick, L.S. Hun, L.M. Young and N. Ritsuko, 2000. Some classes of operators related to paranormal and log-hyponormal operators. Math. Jap., 51: 395-402.

Rashid, M.H.M. and H. Zguitti, 2011. Weyl type theorems and class $\mathrm{A}(\mathrm{s}, \mathrm{t})$ operators. Math. Ineq Appli., 14: 581-594.

Tanahashi, K., 1999. On log-hyponormal operators. Integ. Eq. Operator Theory, 34: 364-372. DOI: 10.1007/BF01300584

Uchiyama, A. and K. Tanahashi, 2002. On the riesz idempotent of class $A$ operators. Math. Ineq. Appl., 5: 291-298.

Uchiyama, A., 2001. weyl's theorem for class $A$ operators. Math. Ineq. Appli., 4: 143-150.

Uchiyama, A., K. Tanahashi and J.I. Lee, 2004. Spectrum of class A (s, t) operators. Actasci Math., (Szeged), 70: 279-298.

Wang, D. and J.I. Lee, 2003. Spectral properties of class a operators. Trends Math. Inform. Center Math. Sci., 6: 93-98.

Yamazaki, T., M. Ito and T. Furuta, 1999. A subclass of paranormal including class of log-hyponormal and several related classes. Sci. Math., 1: 41-45.

Yanagida, T., 2000. On powers of class $\mathrm{A}(k)$ operators including $p$-hyponormal and log-hyponormal operators. Math. Ineq. Appli., 3: 97-104.

Yoshino, T., 1997. The $p$-Hyponormality of The Aluthge Transform. Interdisciplinary Inform. Sci., 3: 91-93. 